\title{
Activation of DNA Damage Signaling Components by Diagnostic Computed Tomography (CT) Scans Detected in Patient Samples Using an Electrochemiluminescence-Based Assay Platform
}

\author{
Yiching Hseih', Ulrike Begley', Lauren Endres², James Keith' ${ }^{1}$, Antonietta F. Hansen², \\ Laurence Kaminsky², Brian McCandless ${ }^{2}$, Thomas J. Begley ${ }^{*}$ \\ ${ }^{1}$ Colleges of Nanoscale Science and Engineering, State University of New York Polytechnic Institute, Albany, NY, USA \\ ${ }^{2}$ College of Arts and Sciences, Stratton Veterans Administration Medical Center, State University of New York Polytechnic \\ Institute, Albany, NY, USA \\ Email: ^tbegley@sunypoly.edu
}

How to cite this paper: Hseih, Y., Begley, U., Endres, L., Keith, J., Hansen, A.F., Kaminsky, L., McCandless, B. and Begley, T.J. (2017) Activation of DNA Damage Signaling Components by Diagnostic Computed Tomography (CT) Scans Detected in Patient Samples Using an Electrochemiluminescence-Based Assay Platform. Advances in Bioscience and Biotechnology, 8, 228247.

https://doi.org/10.4236/abb.2017.87017

Received: May 25, 2017

Accepted: July 24, 2017

Published: July 27, 2017

Copyright ( 92017 by authors and Scientific Research Publishing Inc. This work is licensed under the Creative Commons Attribution International License (CC BY 4.0).

http://creativecommons.org/licenses/by/4.0/ (c) (i) Open Access

\begin{abstract}
Technologies that measure activation of components of the DNA damage response (DDR) have applications in exposure assessment and personalized medicine. The DDR and associated DNA repair pathways encompass hundreds of proteins, making detailed measurement of activation technically challenging and laborious. The purpose of our study was to develop proteinspecific assays for certain DDR components on a high-throughput electrochemiluminescence (ECL)-based platform. We developed five working assay pairs for ataxia telangiectasia mutated (ATM), checkpoint kinase 2 (CHK2), phosphorylated-ATM S1981, phosphorylated-CHK2 T68 and phosphorylatedtumor protein $\mathrm{p} 53$ (p53) S15. We validated the ECL results against traditional immunoblot and $\gamma$-H2AX foci measures in cell and cancer models. In an effort to test the ECL-based technology in a clinical setting, we utilized peripheral blood mononuclear cells (PBMCs) from patients undergoing computed tomography (CT) scans. CT scans represent both a valuable medical imaging diagnostic and a controlled environmental exposure to ionizing radiation for research studies, as they deliver $\sim 2$ to 31 millisieverts $(\mathrm{mSv})$ and are known to activate DDR components. In this study, we show that ECL-based technology can measure the basal and damage-induced levels of DDR components in patient PBMC samples. Using a blinded study design and patient matched preand post CT scan samples, we show that ECL-derived data can consistently
\end{abstract}


(94\% of the time, 15/16 patients) identify PBMCs that have been exposed to low dose ionizing radiation associated with CT scans. Ultimately, the results of our pilot clinical study support the idea that ECL-based technology is applicable for use in clinical and population cohorts that study components of the DDR.

\section{Keywords}

DNA Damage Response, Ionizing Radiation, Diagnostic CT-Scan, Clinical Samples, ATM, CHK2, p53

\section{Introduction}

Ionizing radiation (IR) promotes the formation of single and double DNA strand breaks by directly damaging the DNA molecule and by generating reactive oxygen species that damage DNA. Both the IR-induced DNA damage and subsequent DNA repair contribute to strand breaks [1] [2] [3] [4] [5]. DNA strand breaks promote the regulation of proteins associated with the DNA damage response (DDR), which includes damage-sensor, damage-transducer and DNA repair systems [6] [7] [8]. Ataxia telangiectasia mutated (ATM) is activated by IR to signal the presence of DNA damage [9]. ATM is a serine/threonine protein kinase which autophosphorylates at serine 1981 (S1981) during the response to DNA strand breaks [10], provoking its recruitment to double strand breaks to phosphorylate the $\mathrm{H} 2 \mathrm{~A}$ histone family member, $\mathrm{X}$ protein (i.e., $\gamma$ $\mathrm{H} 2 \mathrm{AX}$ foci) [11]. In response to double strand breaks, ATM will also phosphorylate checkpoint kinase 2 (CHK2) at threonine 68 (T68) to transduce the damage signal and promote downstream phosphorylation of many other proteins [12]. For example, CHK2 can phosphorylate the phosphatase cell division cycle 25 (CDC25) protein to regulate progression through the cell cycle [13]. DNA strand breaks that activate ATM will also promote the phosphorylation of the tumor suppressor protein 53 (p53) at serine 15 (S15) [9]. ATM, CHK2 and p53 signals also regulate cell cycle progression and promote efficient DNA repair. Notably, deficiencies in ATM, CHK2 or p53 lead to genome instability syndromes, cancer predisposition and increased sensitivity to IR exposure [14]-[23].

While IR can initiate DNA damage, it is also an essential component of medical diagnostic equipment. Computed tomography (CT) scans are radiationbased imaging procedures that have been proven to be valuable clinical tools for the diagnosis of injuries to the lungs, heart, liver, spleen, kidneys and bowel. Side effects of diagnostic CT are exposure-induced DNA damage [24] and increased cancer risk [25], with the latter potentially influenced by an individual's response to DNA strand breaks. It has been estimated that patients undergoing diagnostic CT receive $\sim 2$ millisieverts $(\mathrm{mSv}$ ) of radiation for a routine head $\mathrm{CT}$, to $31 \mathrm{mSv}$ for abdomen and pelvis CT scans [26]. $31 \mathrm{mSv}$ is a significant amount of radiation, as it has been documented that Hiroshima and Nagasaki atomic bomb survivors who received $10-100 \mathrm{mSv}$ of radiation had increased cancer risk [27] 
[28] [29]. The use of diagnostic CT scans in the United States of America has increased from 3 million scans in 1980 to nearly 70 million in 2007, and it has been estimated that diagnostic CT scans will cause approximately 15,000 cancer deaths annually [25]. Furthermore, published reports indicate that diagnostic CT scans promote the formation of $\gamma$-H2AX foci in peripheral blood mononuclear cells (PBMCs), supporting the theory that diagnostic imaging using ionizing radiation can in fact provoke a DDR at the cellular level in patients [30] [31] [32].

A large portion of the U.S. population will be exposed to diagnostic CT scans in their lifetime. The benefits of diagnostic CT scans greatly outweighs the potential adverse effects at this time, but tools that measure an individual's ability to respond to CT scans and repair DNA strand breaks are important due to their potential to identify patients susceptible to CT scan induced disease. The current challenge is that measuring DDR and repair is extremely difficult, and a single all-encompassing assay does not exist. However, a number of individual assays are available to study specific DNA damage levels or components of the DDR. These assays include the single cell gel electrophoresis assay (COMET) [33] [34] [35], mass spectrometry based quantification of specific DNA lesions [36], host extract based molecular beacons [37], microarray analysis of transcripts [38] and fluorescence-based multiplex flow cytometric host cell reactivation assays (FMHCR) that measure the repair of specific lesions [39] [40] [41]. In general, these assays provide valuable data on the integrity of DNA or parts of the DDR, and each assay has proved to be extremely useful for research studies on the cellular DDR and DNA repair capacity.

The cellular response to DNA damage includes phosphorylation of many sensor, signaling and repair proteins, making post-translational modifications (PTMs) effective biomarkers of DNA damage and indicators of response. High throughput COMET assays, high throughput $\gamma$-H2AX foci measures and multiplexed fluorescent host cell reactivation assays have highlighted the value of measuring multiple components or outputs [41] [42] [43] [44], with protein based measures being a desirable goal. Assays that measure many PTMs and DNA repair proteins have potential uses in basic, clinical and population studies. Immunological analysis of epitopes on specific proteins is a proven low-tech approach for analyzing proteins and can be accomplished using immunoblots and enzyme-linked immunosorbent assays (ELISA), and such approaches are the work-horses of most biomedical research and diagnostic labs. Unfortunately, immunoblots and ELISA are not readily applicable to the simultaneous and quantitative analysis of the hundreds of proteins and activated targets associated with the DDR, as they suffer from throughput and dynamic range issues. A developed electrochemiluminescence (ECL) technology platform (from MesoSale Discovery, MSD, Gaithersburg, Maryland) is available for the analysis of many $(\sim 3840)$ proteins/targets from the same sample, representing a technology that could be applied to the problem of measuring individual DDR capacity, since its multi-target approach and enhanced sensitivity overcomes many of the afore- 
mentioned issues associated with conventional immunoblot/ELISA assays [45]. The system is best described as ECL detection of an antibody captured protein using an electrode-lined microplate to initiate the signal and sensitive optics used to detect the signal. Each well-specific electrode can be coated and linked to a capture antibody, with the capture antibody used to bind a specific sample protein (i.e., the analyte). Once the capture antibody binds the target protein, the well is washed to remove non-specifically bound proteins. Next, a second antibody that is specific to a different epitope on the target protein is added. Importantly, this second antibody is labeled with a sulfo-tag. The ECL reaction is only initiated if the sulfo-tag labeled antibody binds the target protein, as the label will be stimulated to emit light if electricity is passed from the electrode through the bound protein. Importantly, this design helps reduce background signal as the stimulation (electricity) is decoupled from the signal (light) and it provides a wide dynamic range [46] [47] [48]. The drawback of the MSD-ECL technology platform is that it is only as good as the target-specific capture and detection antibodies, which require extensive development and testing.

The main purpose of our study was to identify commercially available antibodies, specific to DDR proteins, which would work effectively in the ECL platform. In addition, we wanted to compare the performance of the ECL platform to standard measures of an activated DDR, and determine if the platform was amenable for use with clinical samples. As such, we have identified 10 commercially available DDR compatible antibodies for use in the above described MSD-ECL technology platform. Using cell culture models and exposure conditions that promote DNA damage (relative to unexposed, base-line, conditions), we have identified antibodies to ATM, CHK2, phosphorylated-ATM S1981, phosphorylated-CHK2 T68 and phosphorylated-p53 S15 that can be used in the MSD-ECL platform to detect activation of the DDR. Importantly, we have validated the clinical utility and suitability of the ECL-based DDR assays using PBMC samples from patients undergoing diagnostic CT scans. We have demonstrated that the ECL-based technology can identify significant protein level changes induced in vivo by diagnostic CT scans.

\section{Material and Methods}

\subsection{Cell Culture and Treatments}

The human embryonic kidney cell line HEK-293G was purchased from Invitrogen (Grand Island, NY). The human breast and cancer breast cell lines MCF10A, MCF7, T47D, SW527 and MDA-MB231 were kindly provided by Dr. Ceshi Chen (Albany Medical College, Albany, NY). HEK-293G, MDA-MB231 and SW527 were cultured in Dulbecco's Modified Eagle's Medium (Hyclone, South Logan, UT) supplemented with 10\% FBS (Sigma-Aldrich, St. Louis, MO), 100 units $/ \mathrm{ml}$ penicillin and $100 \mathrm{mg} / \mathrm{ml}$ streptomycin (Hyclone) at $37^{\circ} \mathrm{C}$ in $5 \% \mathrm{CO}_{2}$ humidified air. MCF10A cells were cultured in F12/DMEM media (Hyclone) with $5 \%$ horse serum (Invitrogen), 100 units $/ \mathrm{ml}$ penicillin and $100 \mathrm{mg} / \mathrm{ml} \mathrm{strep-}$ tomycin (Hyclone), $20 \mathrm{ng} / \mathrm{ml}$ EGF (Invitrogen), $100 \mathrm{ng} / \mathrm{ml}$ cholera toxin (Sigma- 
Aldrich), $0.01 \mathrm{mg} / \mathrm{ml}$ insulin (Invitrogen) and $500 \mathrm{ng} / \mathrm{ml}$ hydrocortisone (Sigma-Aldrich) at $37^{\circ} \mathrm{C}$ in $5 \% \mathrm{CO}_{2}$ humidified air. MCF7 and T47D cells were cultured in Eagle's MEM (Hyclone) with 10\% FBS (Sigma-Aldrich), 100 units $/ \mathrm{ml}$ penicillin and $100 \mathrm{mg} / \mathrm{ml}$ streptomycin (Hyclone) and $0.01 \mathrm{mg} / \mathrm{ml}$ insulin (Invitrogen) at $37^{\circ} \mathrm{C}$ in $5 \% \mathrm{CO}_{2}$ humidified air. For treatment with chemicals, cells were cultured in medium with bleomycin (Research Products International, Mount Prospect, IL), etoposide (MP Biomedicals, Santa Ana, CA) or $\mathrm{H}_{2} \mathrm{O}_{2}$ (ACROS Organics, Geel, Belgium) at concentrations indicated in the figure legends. Ionizing radiation was delivered by a Faxitron X-ray generator (Faxitron, Tucson, AZ). Cells were irradiated and harvested 1 hour after exposure.

\subsection{Antibodies}

All antibodies used for ECL-based detection and immunoblotting are shown in Supplemental Table S1. The order of addition for ECL-based detection and the concentrations used are also described in Supplemental Table S1.

\subsection{Cell Staining and Imaging Flow Cytometry}

Cells were fixed with $2 \%$ paraformaldehyde for 30 minutes on ice followed by permeabilization and staining with an anti- $\gamma$-H2AX antibody, directly conjugated to FITC (Millipore, Billerica, MA) in PBS containing 0.1\% Triton X-100 for 1 hour on ice. After two washes with PBS, cells were counterstained with 5 mM DRAQ5 (Cell Signaling, Danvers, MA) and analyzed using an ImageStream imaging flow cytometer (Amnis, Seattle, WA). 10,000 cells were collected for each sample and analyzed using IDEAS v4.0 software (Amnis). To quantify the $\gamma$-H2AX foci, single focused cells were gated and the number of foci in each cell was calculated by the spot count wizard algorithm, provided by the IDEAS software.

\subsection{Protein Extraction}

Whole cell protein extracts were prepared with lysis buffer $(20 \mathrm{mM}$ Tris-Cl, $\mathrm{pH}$ 7.4, $150 \mathrm{mM} \mathrm{NaCl}, 1 \mathrm{mM}$ EDTA, 1\% Triton X-100) supplemented with freshly added complete protease inhibitor (Roche Applied Science, Nutley, NJ), phosphatase inhibitor cocktail 2 (Sigma-Aldrich) and phosphatase inhibitor cocktail 3 (Sigma-Aldrich). Protein concentrations were determined using the Bradford protein assay kit (BioRad, Hercules, CA).

\subsection{Immunoblotting}

Whole cell lysates were resolved on SDS-PAGE and then blotted onto PVDF membranes (Millipore). After blocking with 5\% nonfat dried milk in TBS-T (20 $\mathrm{mM}$ Tris- $\mathrm{HCl} \mathrm{pH}$ 7.4, $150 \mathrm{mM} \mathrm{NaCl}, 0.05 \%$ Tween-20) for 1 hour, membranes were incubated with specific primary antibodies in TBS-T with 3\% BSA (SeraCare Life Sciences, Milford, MA) at $4^{\circ} \mathrm{C}$ overnight. After the membrane was washed 4 times with TBS-T, it was incubated for 1 hour at room temperature with the appropriate horseradish peroxidase-conjugated secondary antibody 
(Pierce/Thermo Fisher, Grand Island, NY) prepared with 3\% nonfat milk in TBS-T. The membrane was washed 4 times with TBS-T before protein detection. Proteins were detected using an ECL western blotting substrate (Pierce/Thermo Fisher) and the Fluorchem M imaging system (Protein Simple, Santa Clara, CA).

\subsection{ECL-Based Analysis of DDR Targets}

96-well plates (MesoScale Discoveries, Rockland, MA) were coated with capture antibodies diluted in PBS at $4^{\circ} \mathrm{C}$ overnight. After the plates were washed with the MSD wash buffer (TBS containing 0.2\% Tween-20), plates were blocked with a $5 \%$ blocker A (BSA) (SeraCare Life Sciences) solution at room temperature for 1 hour. Samples were then added to the wells, incubated at $4^{\circ} \mathrm{C}$ overnight or at room temperature for 2 hours. After sample incubation, the plate was washed with wash buffer and the detection antibody (MesoScale Discoveries) was then added to the plates and incubated for 2 hours. Following a further wash with the MSD wash buffer, sulfo-tag anti-species antibodies (MesoScale Discoveries) were then added to each well $(0.5 \mathrm{mg} / \mathrm{ml})$ and the plates were incubated for 1 hour at room temperature. Finally, plates were washed with the MSD wash buffer, and MSD read buffer $\mathrm{T}$ was added to detect the signal of analyte using the Sector 2400 instrument (MesoScale Discoveries).

\subsection{Chicken Chorioallantoic Membrane Assay (CAM Assay)}

Fertilized White Leghorn chicken eggs (Charles River, Cambridge, MA) were initially incubated for 10 days at $37^{\circ} \mathrm{C}$ in a humidified atmosphere inside a hatching incubator equipped with an automatic rotator (Octagon 20, Brinsea, Somerset, UK). Xenografts were then performed as previously described [49]. Briefly, a square window of $\sim 1 \mathrm{~cm}$ was opened over the displaced CAM and sealed with a piece of sterile tape. MDA-MB231 cells were detached from the culture plate with $2 \mathrm{mM}$ EDTA in PBS and washed twice in PBS. Cells $\left(2 \times 10^{6}\right)$ were resuspended in $50 \mu \mathrm{PBS}$ containing $1 \mathrm{mM} \mathrm{MgCl}, 0.5 \mathrm{mM} \mathrm{CaCl}_{2}, 100 \mathrm{U} / \mathrm{ml}$ penicillin and $100 \mu \mathrm{g} / \mathrm{ml}$ streptomycin and then inoculated on the CAM. The opening was re-sealed with tape and the eggs were placed in a stationary incubator at $37^{\circ} \mathrm{C}$ for 7 days or as indicated. The resulting tumors were removed, photographed and proteins were analyzed as described above.

\subsection{Blood Draw and PBMC Isolation}

After providing informed consent, whole blood samples were collected from subjects prior to, and 1 hour post CTscan for the purpose of clinical diagnosis. Blood was stored de-identified in glass plasma tubes (143 USP) containing freeze-dried sodium heparin (Beckton, Dickinson and Company, Franklin Lakes, NJ). PBMCs were then isolated with Histopaque-1077 (Sigma-Aldrich, St. Louis, MO) following manufacturer's instructions. Briefly, whole blood samples were diluted with RPMI medium at a 2:1 ratio and then layered on top of $15 \mathrm{ml}$ Histopaque-1077. Samples were centrifuged at $400 \times \mathrm{g}$ for 30 minutes at room 
temperature and the buffy coat layer, containing mostly white blood cells, was removed and transferred into a different centrifuge tube. Cells were washed 3 times with the original volume of PBS, and then centrifuged at $250 \times \mathrm{g}$ for 10 minutes at room temperature. The wash step was repeated and cell pellets were then stored at $-80^{\circ} \mathrm{C}$.

\section{Results and Discussion}

\subsection{IR Induced Activation of DDR as Measured by $\gamma-\mathrm{H} 2 \mathrm{AX}$ Foci Formation, Immunoblot- and ECL-Analysis of Cell Culture Models}

The ATM-CHK2-p53 pathway is activated in response to DNA double strand breaks induced by IR [6] [9] [10] [11] [12]. To support that the irradiated cells used in our study had DNA double strand breaks and an activated DDR, we stained IR-exposed HEK-293G cells with $\gamma$-H2AX antibody and analyzed foci formation using imaging flow cytometry (Figure 1(a)). We observed a marked increase in $\gamma$-H2AX foci in the irradiated HEK-293G cells (8 Gy), relative to unexposed control cells. To further examine IR-induced activation of the DDR, we measured specific protein and post-translational modification levels pre- and post-IR exposure in HEK-293G cells. As shown by immunoblotting in Figure 1(b), phosphorylated ATM S1981, phosphorylated CHK2 T68 and phosphorylated p53 S15 were markedly increased after IR exposure.

Next we focused on the use of DDR markers as initial targets for multiplexed protein quantification assays using MSD's ECL technology platform. As shown in Figure 1(c), each antibody sandwich assay was initiated by coating with a specific capture antibody that recognizes the targeted protein. After blocking, samples were added, followed by the addition of a detection antibody that recognizes the targeted protein or protein-specific modification. Once the sandwich was formed, an anti-species sulfo-tag antibody was added, which when bound emits light upon electrical stimulation. After screening more than 30 antibodies, we developed five working assays (Figure 1(d)) that quantitatively measure IR-induced activation of some DDR components in the HEK-293G cells. Capture antibodies included those for phosphorylated ATM S1981, ATM, phosphorylated CHK2 T68, CHK2 and phosphorylated p53 S15. Consistent with immunoblot results, the mean signals of phosphorylated ATM S1981, phosphorylated CHK2 T68, CHK2 and phosphorylated p53 S15 were increased after cells were exposed to IR, while the levels of total ATM protein remain the same. We have performed similar studies using a wide range of culture systems (HEK293G, MCF10A, MCF7, T47D, SW527 and MDA-MB231) exposed to a range of DNA damaging agents (bleomycin, etoposide, $\mathrm{H}_{2} \mathrm{O}_{2}$ and 4 Gy IR) to further demonstrate the utility of the assay platform (Supplemental Figure S1-S3, Table S2) in detecting activation of the DDR. In addition, we have demonstrated that the ECL-based platform can be used to measure IR-induced activation of the DDR in tumor xenografts (Supplemental Figure S4). 


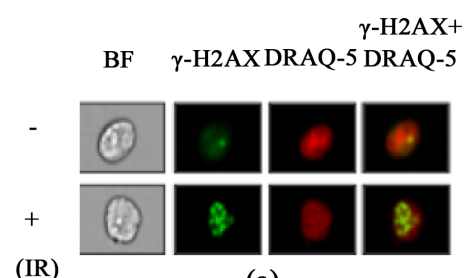

(a)

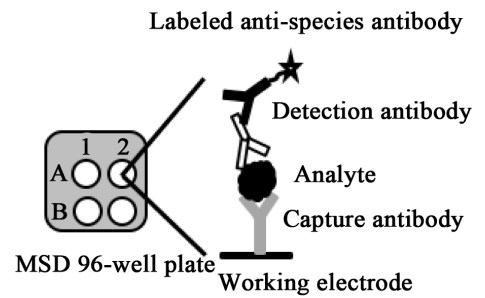

(c)

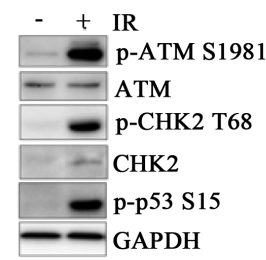

(b)

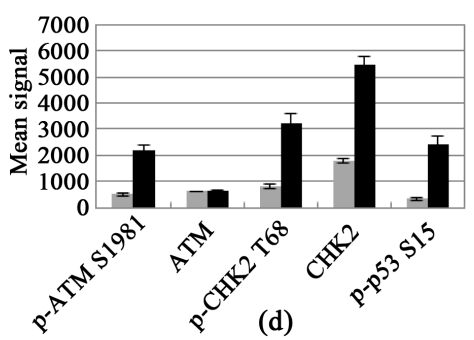

(d)

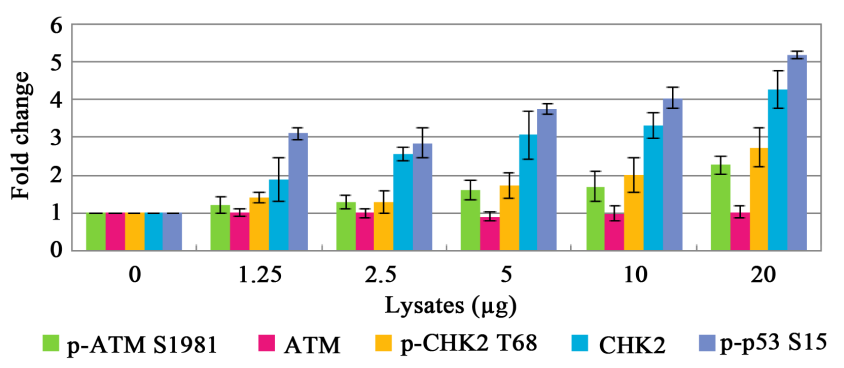

(e)

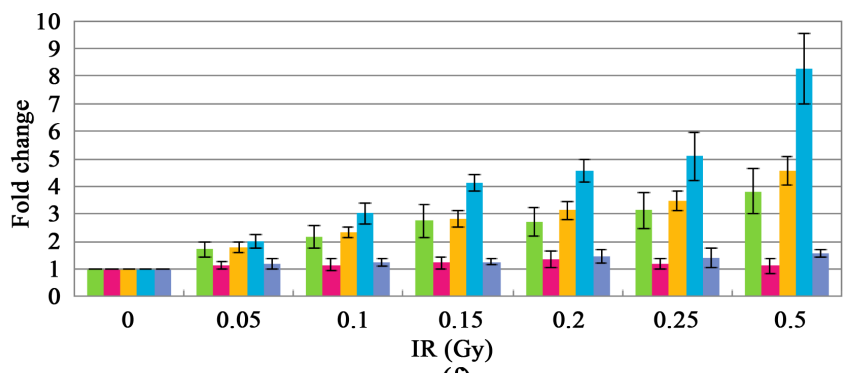

(f)

Figure 1. DDR assay development and IR based validation in HEK-293G cells. HEK-293G cells were left unexposed or irradiated with ionizing radiation (4 Gy). Cells were incubated for 1-hour, fixed and (a) stained with $\gamma$-H2AX-FITC and DRAQ-5, followed by imaging flow cytometry using the Amnis Imagestream; (b) Immunoblot analysis of specific DDR proteins was performed using anti-phosphorylated ATMS1981, anti-ATM, anti-phosphorylated CHK2 T68, anti-CHK2 and anti-phosphorylated p53 S15. GAPDH was used as a loading control; (c) The ECL-based assay design has the target protein captured and detected by antibodies that recognize two different epitopes on a protein. The third labeled anti-species antibody works as a reporter that emits light when electrically stimulated, with the emitted light quantitatively read by the Sector 2400 instrument; (d) HEK-293G cells were left unexposed (grey bars) or irradiated (4 Gy, black bars) and harvested 1-hour after exposure. $20 \mu \mathrm{gs}$ of lysates were added to each well of an MSD MULTI-ARRAY 96-well plate that was pre-coated with specific capture antibodies and analyzed by ECL. The mean $(\mathrm{N}=3)$ and standard deviation are reported; (e) HEK-293G cells were irradiated with ionizing radiation ( $4 \mathrm{~Gy}$ ) and different amounts of lysate (0 to $20 \mathrm{ug}$ ) were assayed as described in panel D; (f) HEK-293G cells were irradiated with ionizing radiation ( $0,0.05$ to $0.5 \mathrm{~Gy})$ and $20 \mu \mathrm{gs}$ of lysate was added to each well and analyzed as described in panel D. 
We also investigated how much input protein was needed to detect IR-induced activation of the DDR. We determined that the ECL-based assay can detect activation of our DDR components with as little as $1.25 \mu \mathrm{g}$ of input sample, compared to the $30 \mu \mathrm{g}$ required for the immunoblotting experiments (Figure 1(e) vs. Figure 1(b)). Notably, we can detect about a 3 -fold increase in phosphorylated p53 S15 signal with $1.25 \mu \mathrm{g}$ of lysates coming from cells irradiated at $8 \mathrm{~Gy}$. Once protein extracts were produced, the assay was performed in under 3-hours, with the plate based design amenable to high throughput analysis. Ultimately our results support the idea that the developed ECL-based assays are a sensitive, quantitative and fast way to measure the IR-induced activation of DDR components in HEK-293G cells.

We also investigated if the ECL-based approach could detect activation of the DDR after low dose exposure to IR. We decreased the IR dose from 4 to 0.5 $0.05 \mathrm{~Gy}$ and determined whether the ECL based methodology could detect activation of the DDR. 1hour after HEK-293G cells were exposed to 0.05 Gy of IR, we detected $\sim 2$-fold increases in the levels of phosphorylated ATM S1981 and phosphorylated CHK2 T68, with phosphorylated p53 S15 levels showing little change (Figure 1(f)). 1 hour after HEK-293G cells were exposed to 0.1 to 0.5 Gy of IR we could detect further increases in the levels of phosphorylated ATM S1981 (up to $\sim 4$-fold) and phosphorylated CHK2 T68 (up to $\sim 5$-fold). We observed little change in the levels of phosphorylated p53 S15 when cells were exposed to 0.05 to 0.015 Gy of IR (Figure 1(f)). Our results using cells in culture demonstrate that the ECL-based assay specific to DDR components can be used with cell lines and laboratory exposures to detect activation of the DDR, even at relatively low IR doses (0.05 Gy). It also begged the question of whether our ECL-based assays could be used to analyze or detect activation of the DDR in humans exposed to low dose ionizing radiation.

\subsection{Design and Goals of Pilot Clinical Study}

Diagnostic CT scans can deliver 2 to $31 \mathrm{mSv}$ of ionizing radiation [26]. Patients undergoing $\mathrm{CT}$ scans provide a unique population with a well-controlled exposure to a DNA damaging agent, which is a useful setting for testing our ECLbased assay specific to the DDR. Thus we set up a pilot clinical study (Figure 2) to quantitate DDR protein levels in patient PBMC's before and after a diagnostic CT scan. Specifically, we wanted to determine if (1) the ECL-based assay we developed for some DDR components can identify significant protein level changes induced in vivo by diagnostic CT scan and (2), using blinded samples, can the resulting patient-matched data be used to predict whether samples were pre- or post-CT scan samples. Both results would advise us on the suitably of our assay system for use in larger clinical and population studies.

For this pilot study, we recruited men of any ethnicity between the ages of 18 to 75 with appointments at the Stratton Veterans Administration Hospital (Albany, NY) for a diagnostic CT scan of the chest or abdomen/pelvis, as these procedures use similar amounts of ionizing radiation. Enrolled patients were 


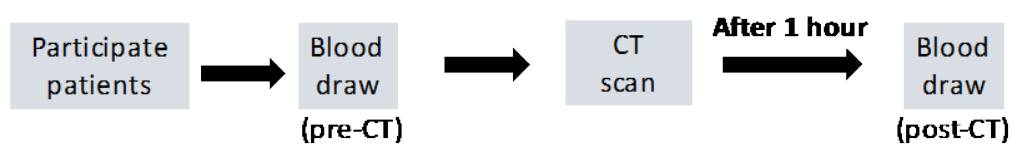

The pre-CT and post-CT blood was supplied as blinded " $\mathrm{A}$ " or " $\mathrm{B}$ " samples, the peripheral blood mononuclear cells were isolated from each and DNA damage response markers were quantitated using the ECL-based assay platform

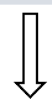

Study Goals

1. Identify pre- and post-CT sample from each patient.

2. Determine if there are patient specific responses to CT-scan.

3. Demonstrate robust technology platform.

Figure 2. Study Design. Participated patients donated $30 \mathrm{ml}$ of whole blood each before and after a diagnostic CT scan. Blood was collected in a sodium heparin glass tube and stored at $4^{\circ} \mathrm{C}$. Blood was transferred from the clinical to research team as blinded patient samples (A or B) that were specific to pre- and post CT scan samples. PBMCs were isolated within 3 hours of post-CT blood draw and stored at $-80^{\circ} \mathrm{C}$. Protein was isolated from all supplied samples at the same time and analyzed for the five markers described in Figure 1.

limited to those having CT scans without the use of intravenous contrast, which could possibly represent a confounding variable. Non-contrast CT is typically ordered to help in the diagnosis of pneumonia, re-evaluation of pulmonary nodules, pleural effusions, adrenal enlargement, abdominal aortic aneurysm and renal calculi. We excluded women from this pilot study because the majority (93\%) of patients at the Stratton Veterans Administration Hospital are men and the inclusion of women would dramatically increase the time needed to recruit subjects, which would not be feasible in the 2-year time span of the associated grant (NIH R21 ES019492). CT is commonly used for diagnostic work in hospitals throughout the world and all recruited subjects were undergoing a clinically prescribed CT scan. Blood was drawn from 16 subjects over the course of 6 months both before and approximately 1 hour after diagnostic CT. Blood was stored on ice, patient specific pre- and post-CT samples were randomly coded by the research coordinator as either patient specific A or B samples. Blood was delivered as blinded samples to research scientists for processing. PBMCs were purified from the blood and stored at $-80^{\circ} \mathrm{C}$ within 3 hours of blood draw, with all samples processed for protein analysis at the same-time. Our study complied with the Declaration of Helsinki and all work was performed under VA and SUNY Institutional Review Board Approval.

\subsection{Significant Difference in Marker Levels in Patient Matched A and B Samples}

We obtained enough protein from each patient's A and B sample to obtain three technical replicates for each analyzed DDR marker, with resulting data reported 
as the average amount (-/+ standard deviation) of phosphorylated ATM S1981, ATM, phosphorylated CHK2 T68, CHK2 and phosphorylated p53 S15 (Figure 3). The Student's t-test was used to determine if differences were significant ( $\mathrm{p}<$ 0.05) in the levels of each marker when comparing a patient's A and B samples (Figure 3 and Table 1). Based on past experience and Figure 1 data, ATM levels do not significantly change after exposure to IR, and for the majority of patient-

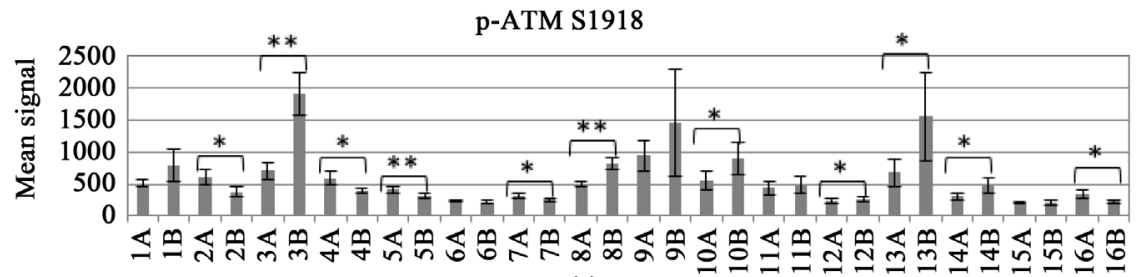

(a)

ATM

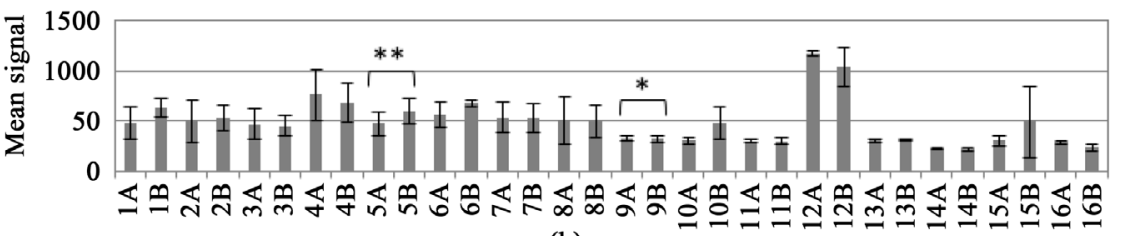

(b)

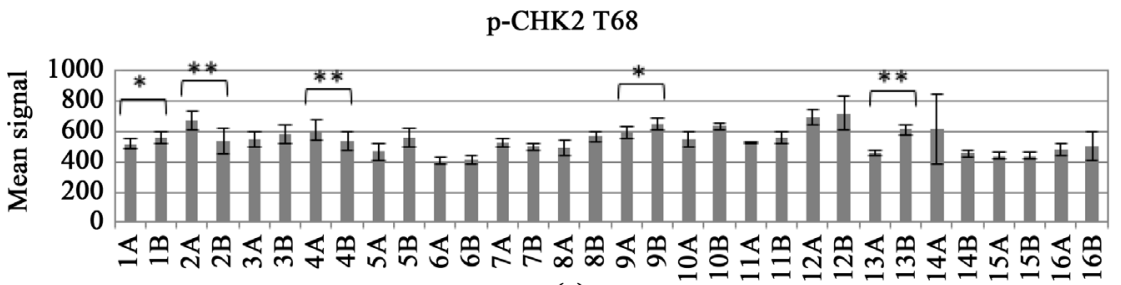

(c)

CHK2

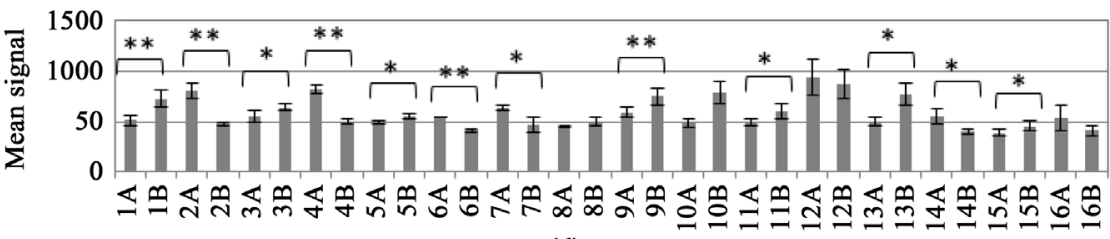

(d)

p-p53 S15

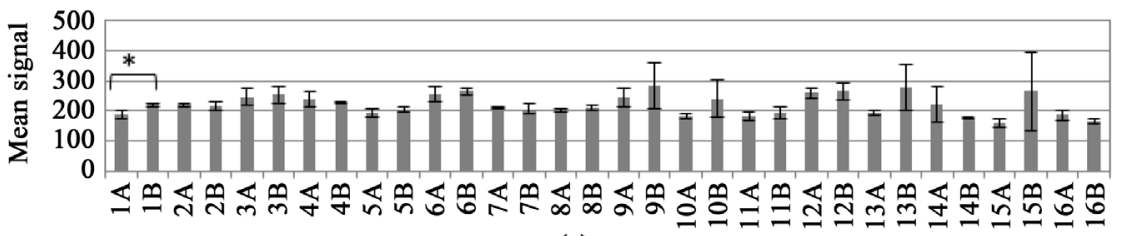

(e)

Figure 3. Intensity of five markers from A and B samples of 16 participated patients. ((a)-(e) Patient specific protein extracts $(25 \mu \mathrm{gs})$ were analyzed $(\mathrm{N}=3)$ for DDR markers using the Figure 1 described ECL-approach. A and B are the blinded codes for either preor post-CT samples for each patient. Significant differences between each patients A and B sample were determined using the Student's t-test $\left({ }^{\star} p<0.05,{ }^{* *} p<0.01\right)$. 
Table 1. Statistical difference between DDR markers in " $A$ " and "B" samples of participated patients. The Student's t-test was used to generate $\mathrm{p}$-values detailing marker level differences between the $\mathrm{A}$ and $\mathrm{B}$ samples for each patient (mean values and standard deviations for raw intensity values are reported in Supplemental Table S3).

\begin{tabular}{|c|c|c|c|c|c|c|}
\hline Patient & p-ATM & ATM & p-CHK2 & CHK2 & p-P53 & \# significant \\
\hline$\# 1$ & 0.085 & 0.211 & 0.025 & 0.006 & 0.025 & 3 \\
\hline$\# 2$ & 0.010 & 0.404 & 0.004 & 0.005 & 0.352 & 3 \\
\hline$\# 3$ & 0.005 & 0.186 & 0.060 & 0.019 & 0.092 & 2 \\
\hline$\# 4$ & 0.016 & 0.320 & 0.003 & 0.003 & 0.261 & 3 \\
\hline$\# 5$ & 0.008 & 0.001 & 0.141 & 0.043 & 0.172 & 3 \\
\hline$\# 6$ & 0.313 & 0.139 & 0.426 & 0.002 & 0.310 & 1 \\
\hline$\# 7$ & 0.031 & 0.445 & 0.158 & 0.041 & 0.296 & 2 \\
\hline$\# 8$ & 0.006 & 0.452 & 0.053 & 0.057 & 0.251 & 1 \\
\hline$\# 9$ & 0.167 & 0.050 & 0.012 & 0.009 & 0.131 & 3 \\
\hline$\# 10$ & 0.018 & 0.070 & 0.256 & 0.075 & 0.079 & 1 \\
\hline$\# 11$ & 0.055 & 0.495 & 0.219 & 0.017 & 0.150 & 1 \\
\hline$\# 12$ & 0.012 & 0.163 & 0.285 & 0.108 & 0.279 & 1 \\
\hline$\# 13$ & 0.045 & 0.141 & 0.009 & 0.012 & 0.116 & 3 \\
\hline$\# 14$ & 0.022 & 0.305 & 0.190 & 0.021 & 0.175 & 2 \\
\hline$\# 15$ & 0.228 & 0.197 & 0.463 & 0.011 & 0.328 & 1 \\
\hline$\# 16$ & 0.050 & 0.069 & 0.299 & 0.081 & 0.224 & 1 \\
\hline
\end{tabular}

matched samples (i.e., 14 out 16 samples), this held true. Phosphorylated p53 S15 levels showed little change in HEK-293G cells exposed to low dose IR (Figure 1(f)). Similarly, 15 out of the 16 patient matched samples showed no difference in phosphorylated p53 S15 when comparing their A and B samples (Figure 3, Table 1). In contrast to ATM and phosphorylated p53, we detected significant $(p<0.05)$ differences in the levels of phosphorylated ATM S1981 (11 out 16 subjects) and CHK2 (12 out of 16 subjects) in a majority of the subjects when comparing their A and B samples (Figure 3, Table 1). While the differences in CHK2 levels were all less than 2-fold, we did detect up to 3-fold differences in the amount of phosphorylated ATM in some subjects. Lastly we determined significant differences in the levels of phosphorylated CHK2 T68 in 5 of 16 subjects (Figure 3, Table 1), but similar to CHK2 data the observed changes were all less than 2-fold. Also all increases in phosphorylated CHK2 T68 where matched by increases in $\mathrm{CHK} 2$ in the same patient.

\subsection{Metrics Used to Predict Pre- and Post CT-Scan Sample for Each Patient}

Data for all five markers were then analyzed in the context of each patient (Figure 3, Table 1), with the increase in marker levels used to predict the post-ex- posure samples. All 16 patients had a significant change in at least one 
marker, with 6 patients having recorded significant changes in 3 markers, 3 patients having recorded significant changes in 2 markers and 7 patients having a recorded significant change in only 1 marker. IR-induced DNA double strand breaks will lead to increased activation of ATM, CHK2 and p53. As such, increases in phosphorylated ATM S1981, phosphorylated CHK2 T68 and phosphorylated p53 S15 levels should all be indicators of the post-CT scan sample. Also based on our assay design, increased CHK2 levels should be an indicator of the post CT scan sample. The 7 patients for which we recorded only one significant increase all had that increase in CHK2 or phosphorylated ATM S1981, which was then used to predict the post-CT sample from A or B (Table 2). We detected significant $(p<0.05)$ changes in 2 to 3 markers for 9 patients. For these 9 patients, we detected correlated increases (i.e., the significant increases for each marker were observed in just the A or just the B sample) for 6 patients. This correlated data was then used to predict whether sample A or B was collected pre- or post-CT sample. We observed marker increases in opposite samples for 3 patients. For patient \#5 we recorded an increase in ATM and CHK2 in the B sample and increase in phosphorylated ATM S1981 in the A sample. Our validation data reported in Figure 1 supports the idea that total ATM protein levels do not change in response to IR, while phosphorylated ATM S1981 and CHK2 protein levels increase. As the increases were in opposite samples for patient \#5's ATM S1981 and CHK2 levels, we viewed the phosphorylated ATM S1981 data as a more reliable predictor because it had a more significant $p$-value $(p<0.008$ vs. $p<0.043)$. Thus we used the phosphorylated ATM S1981 data to predict the post-CT sample for patient \#5 (Table 2). In patient \#9 we observed a significant increases in ATM in the A sample and significant increases in CHK2 and phosphorylated CHK2 T68 in the B sample. In patient \#9 we used the significant increases in the B sample for phosphorylated CHK2 T68 and CHK2 to predict it as the post-CT scan sample. In patient \#14 we detected increases in phosphorylated ATM S1981 and CHK2 in opposite samples. CHK2 had a more significant increase in total signal relative to phosphorylated ATM S1981, $p<0.021$ and $p<$ 0.022 , respectively, which directed us to use CHK2 as the predictor for the postCT scan sample.

\subsection{4\% Prediction Accuracy Using ATM S1981, CHK2 and CHK2 T68 Markers}

Collectively we used biological and statistical rational to predict the post CT sample for each of the 16 matched patient samples (Table 2). When we un-blinded exposure details and aligned them with our quantitative data and compiled predictions, we determined that we had successfully predicted the post CT sample for 15 of the 16 patients (94\% accuracy) (Table 2). Surprisingly the lone prediction error in our small sample occurred with patient \#4 in which we detected correlated increases in CHK2, phosphorylated ATM S1981 and phosphorylated CHK2 T68 in the A sample relative to B, leading to our incorrect designation of $\mathrm{A}$ as the post $\mathrm{CT}$ scan sample. While $100 \%$ is the desired accuracy 
Table 2. Pre-and post-CT blood sample predictions and CTscan details. Predictions (columns 2 - 6) from individual assays were based on the intensity levels of markers (Figure 3) and p-values listed in Table 1, with the cumulative prediction reported in column 7. Blank indicates a marker that was not informative $(p>0.05)$. The prediction accuracy is listed in columns 7. The time between the end of the CTscan and post blood draw, body area imaged, dose-length-product (DLP) is listed in column 8, abbreviations include abdomen (Abd) and Pelvis (Pel).

\begin{tabular}{|c|c|c|c|c|c|c|c|c|}
\hline Code & p-ATM & ATM & $\mathrm{p}-\mathrm{CHK} 2$ & CHK2 & p-P53 & Prediction & Correct & $\begin{array}{c}\text { Time/Type/DLP } \\
\text { mGy-cm }\end{array}$ \\
\hline $1 \mathrm{~A}$ & & & Pre & Pre & Pre & Pre-CT & \multirow{2}{*}{ Yes } & (90 mins) \\
\hline $1 \mathrm{~B}$ & & & Post & Post & Post & Post-CT & & $/($ Chest $) / 674$ \\
\hline $2 \mathrm{~A}$ & Post & & Post & Post & & Post-CT & \multirow{2}{*}{ Yes } & (64 mins) \\
\hline $2 \mathrm{~B}$ & Pre & & Pre & Pre & & Pre-CT & & $/(\mathrm{Abd} / \mathrm{Pel}) / 1607$ \\
\hline $3 \mathrm{~A}$ & Pre & & & Pre & & Pre-CT & \multirow{2}{*}{ Yes } & (66 mins) \\
\hline $3 \mathrm{~B}$ & Post & & & Post & & Post-CT & & $/(\mathrm{Abd} / \mathrm{Pel}) / 1974$ \\
\hline $4 \mathrm{~A}$ & Post & & Post & Post & & Post-CT & \multirow{2}{*}{ No } & (74 mins) \\
\hline $4 \mathrm{~B}$ & Pre & & Pre & Pre & & Pre-CT & & $/($ Chest $) / 581$ \\
\hline $5 \mathrm{~A}$ & Post & Pre & & Pre & & Post-CT & \multirow{2}{*}{ Yes } & (90 mins) \\
\hline $5 \mathrm{~B}$ & Pre & Post & & Post & & Pre-CT & & $/($ Chest $) / 501$ \\
\hline $6 \mathrm{~A}$ & & & & Post & & Post-CT & \multirow{2}{*}{ Yes } & (75 mins) \\
\hline $6 \mathrm{~B}$ & & & & Pre & & Pre-CT & & $/(\mathrm{Abdo}) / 482$ \\
\hline $7 \mathrm{~A}$ & Post & & & Post & & Post-CT & \multirow{2}{*}{ Yes } & (65 mins) \\
\hline $7 \mathrm{~B}$ & Pre & & & Pre & & Pre-CT & & $/($ Chest $) / 658$ \\
\hline $8 \mathrm{~A}$ & Pre & & & & & Pre-CT & \multirow{2}{*}{ Yes } & (61 mins) \\
\hline $8 \mathrm{~B}$ & Post & & & & & Post-CT & & $/(\mathrm{Abd} / \mathrm{Pel}) / 510$ \\
\hline $9 \mathrm{~A}$ & & Post & Pre & Pre & & Pre-CT & \multirow{2}{*}{ Yes } & (97 mins) \\
\hline $9 \mathrm{~B}$ & & Pre & Post & Post & & Post-CT & & $/($ Chest $) / 669$ \\
\hline $10 \mathrm{~A}$ & Pre & & & & & Pre-CT & \multirow{2}{*}{ Yes } & (60 mins) \\
\hline $10 \mathrm{~B}$ & Post & & & & & Post-CT & & $/($ Chest $) / 898$ \\
\hline $11 \mathrm{~A}$ & & & & Pre & & Pre-CT & \multirow{2}{*}{ Yes } & (40 mins) \\
\hline $11 \mathrm{~B}$ & & & & Post & & Post-CT & & $/(\mathrm{Abd} / \mathrm{Pel}) / 476$ \\
\hline $12 \mathrm{~A}$ & Pre & & & & & Pre-CT & \multirow{2}{*}{ Yes } & (73 mins) \\
\hline $12 \mathrm{~B}$ & Post & & & & & Post-CT & & $/(\mathrm{Abd} / \mathrm{Pel}) / 340$ \\
\hline $13 \mathrm{~A}$ & Pre & & Pre & Pre & & Pre-CT & \multirow{2}{*}{ Yes } & (110 mins) \\
\hline $13 \mathrm{~B}$ & Post & & Post & Post & & Post-CT & & $/($ Chest)/989 \\
\hline $14 \mathrm{~A}$ & Pre & & & Post & & Post-CT & \multirow{2}{*}{ Yes } & (65 mins) \\
\hline $14 \mathrm{~B}$ & Post & & & Pre & & Pre-CT & & $/(\mathrm{Abd} / \mathrm{Pel}) / 2207$ \\
\hline $15 \mathrm{~A}$ & & & & Pre & & Pre-CT & \multirow{2}{*}{ Yes } & (70 mins) \\
\hline $15 \mathrm{~B}$ & & & & Post & & Post-CT & & $/($ Chest)/524 \\
\hline $16 \mathrm{~A}$ & Post & & & & & Post-CT & \multirow{2}{*}{ Yes } & (70 mins) \\
\hline $16 \mathrm{~B}$ & & Pre & & & & Pre-Ct & & /(Abd/Pel)/904 \\
\hline
\end{tabular}


for using measures of DDR and DNA repair in clinical and population cohorts, the $94 \%$ value is comparable to other studies. For example, Fry et al., used genomic approaches and computational models to predict susceptibility to DNA damaging agents with $94 \%$ accuracy [38].

\section{Conclusions}

Radiation-based imaging technologies that include CT scans and digital mammography have been reported to activate the DDR [30] [31] [32]. Most studies that have analyzed the biological effect of radiation-induced imaging have done this by quantitating the formation of $\gamma-\mathrm{H} 2 \mathrm{AX}$ foci in the cell by fluorescent microscopy. Our study is unique in that we quantitated the levels of five different components of the DDR, with ATM being the kinase that is used to phosphorylate $\mathrm{H} 2 \mathrm{AX}$. We have shown that the ECL based quantification can identify significant protein level changes induced by diagnostic CT scan. Our data supports the idea that quantitative analysis of multiple DDR components was the best predictor of exposure to the low level ionizing radiation delivered by diagnostic CTscan (Supplemental Table S4). For example, using phosphorylated ATM or phosphorylated CHK2 individually, the prediction accuracy was 50\%-56\%, but using together with $\mathrm{CH} 2 \mathrm{~K}$ and phosphorylated p53 the use of four markers increased the accuracy to $94 \%$. Simply put, the use of multiple markers provided the best accuracy and, in many cases, provided redundancy in predicting the post CT scan sample.

One interesting observation specific to phosphorylated ATM S1981 was that three groups of patients with different levels of this marker were observed after CT scan (Figure 4). Specifically, we identified groups of patients that had low, medium and high levels of phosphorylated ATM S1981 that were significantly

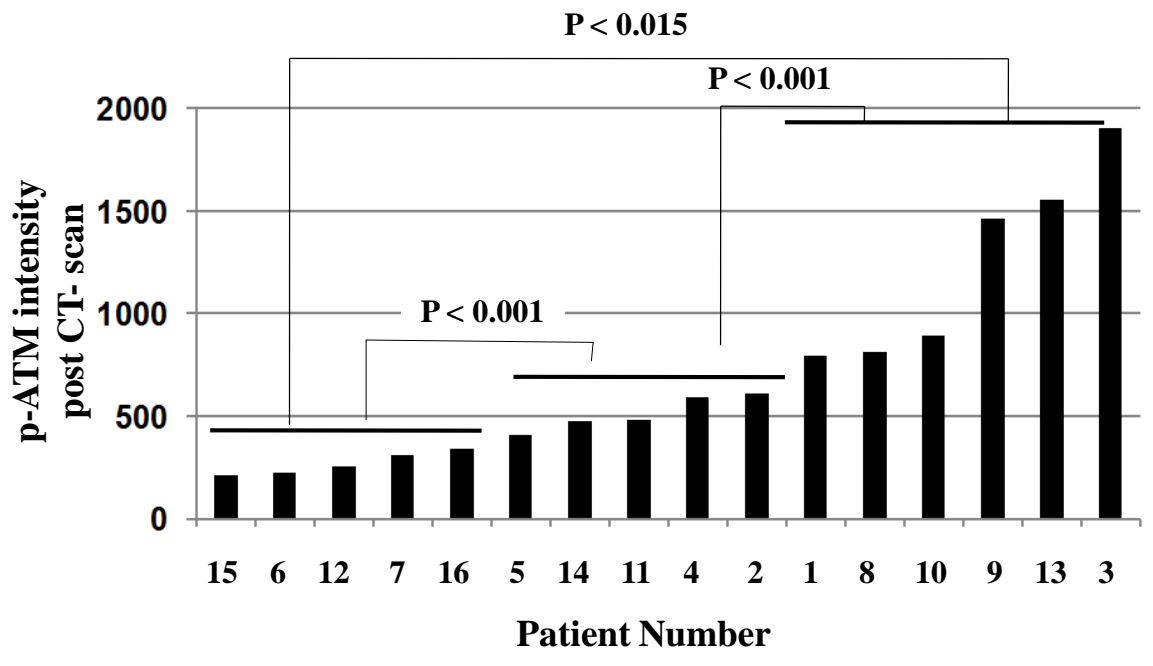

Figure 4. Stratification of patients based on post CT scan protein levels. The absolute level of phosphorylated ATM from each of 16 patients was ordered from low to high. Groups of patients were compared using the Student's t-test, to identify low, medium and high designates. Student's t-test was used to assess for significant differences in the low, medium and high groups. 
$(p<0.05)$ different from one another. These findings suggest that there are differential responses to a CT scan in the population, but future experiments are needed to address this possibility and any potential underlying mechanisms. For instance, individuals may vary in their ability to mount and sustain a protective response to ionizing radiation, particularly at diagnostic levels, perhaps due to uncharacterized genetic variation in one (or more) components of the DDR. In theory, these individuals could be at a greater risk for CT scan induced disease, and this risk could be mitigated if an individual's DDR capacity is factored into decisions about diagnostic imaging.

Increased sensitivity to diagnostic $\mathrm{CT}$ in specific people, as marked by the persistence of $\gamma$-H2AX foci, has been reported and was most likely due to a defect in double strand break repair [50]. Lobrich et al. report that most patients respond to diagnostic CT's by forming $\gamma$-H2AX foci, with these usually falling to baseline levels 24-hours after the procedure. One notable exception was a patient whom displayed high levels of CT scan induced $\gamma$-H2AX foci 24-hours post exposure and could have a genome instability syndrome. Genetic factors have also been shown to dramatically affect the response to DNA damage. In cell culture models specific to the Coriell collection, which are lymphoblastoid cell lines from 460 unrelated individuals that reflect the diversity of the human population [51], Fry et al., have demonstrated inter-individual differences in the cellular response to alkylating agents. These differences occur in the transcriptional response and cell viability post-treatment with an alkylating agent, with their study identifying transcriptional signatures that classify cells sensitive or resistant to killing by methylnitronitrosoguanidine (MNNG) [38]. The identified inter-individual differences identified by microarray analysis have application in cancer treatment, as MNNG is representative of some alkylation based chemotherapeutics. Our pilot study also demonstrates inter-individual variability in the response to diagnostic CTs, with patients showing increases in anywhere from one to four marker levels. Notably we have demonstrated that tumors grown from xenografts can be analyzed using our ECL-based assay, which suggests applications in cancer treatment.

Ultimately, the ECL based assay platform we used proved to be highly reliable, providing tight technical replicates for each of the five DDR targets. Our study supports the idea that ECL is a viable technology platform for use with clinical studies. The ECL assay platform has the potential to be expanded to $>3450$ samples. With over two hundred proteins associated with the DDR and many being post-translationally modified, the ECL technology platform could completely cover the DDR protein space. Increasing the number of DDR proteins that can be analyzed by the ECL-based platform is a future goal.

\section{Acknowledgements}

Financial support was provided by the National Institute of Environmental Health Sciences (R21 ES019492). 


\section{References}

[1] Ma, W., et al. (2009) The Transition of Closely Opposed Lesions to Double-Strand Breaks during Long-Patch Base Excision Repair Is Prevented by the Coordinated Action of DNA Polymerase Delta and Rad27/Fen1. Molecular and Cellular Biology, 29, 1212-1221. https://doi.org/10.1128/MCB.01499-08

[2] Friedberg, E., et al. (2005) DNA Repair and Mutagenesis. 2nd Edition, ASM Press.

[3] Ward, J.F. (1985) Biochemistry of DNA Lesions. Radiation Research Supplement, 8, S103-S111. https://doi.org/10.2307/3583517

[4] Riley, P.A. (1994) Free Radicals in Biology: Oxidative Stress and the Effects of Ionizing Radiation. International Journal of Radiation Biology, 65, 27-33. https://doi.org/10.1080/09553009414550041

[5] Ward, J.F. (1990) The Yield of DNA Double-Strand Breaks Produced Intracellularly by Ionizing Radiation: A Review. International Journal of Radiation Biology, 57, 1141-1150. https://doi.org/10.1080/09553009014551251

[6] Zhou, B.B. and Elledge, S.J. (2000) The DNA Damage Response: Putting Checkpoints in Perspective. Nature, 408, 433-439. https://doi.org/10.1038/35044005

[7] Elia, A.E., et al. (2015) Quantitative Proteomic Atlas of Ubiquitination and Acetylation in the DNA Damage Response. Molecular Cell, 59, 867-881.

[8] Bensimon, A., et al. (2010) ATM-Dependent and -Independent Dynamics of the Nuclear Phosphoproteome after DNA Damage. Science Signaling, 3, rs3.

https://doi.org/10.1126/scisignal.2001034

[9] Canman, C.E., et al. (1998) Activation of the ATM Kinase by Ionizing Radiation and Phosphorylation of p53. Science, 281, 1677-1679. https://doi.org/10.1126/science.281.5383.1677

[10] Bakkenist, C.J. and Kastan, M.B. (2003) DNA Damage Activates ATM through Intermolecular Autophosphorylation and Dimer Dissociation. Nature, 421, 499-506. https://doi.org/10.1038/nature01368

[11] Burma, S., Chen, B.P., Murphy, M., Kurimasa, A. and Chen, D.J. (2001) ATM Phosphorylates Histone H2AX in Response to DNA Double-Strand Breaks. The Journal of Biological Chemistry, 276, 42462-42467. https://doi.org/10.1074/jbc.C100466200

[12] Matsuoka, S., et al. (2000) Ataxia Telangiectasia-Mutated Phosphorylates Chk2 in Vivo and in Vitro. Proceedings of the National Academy of Sciences of the United States, 97, 10389-10394. https://doi.org/10.1073/pnas.190030497

[13] Falck, J., Mailand, N., Syljuasen, R.G., Bartek, J. and Lukas, J. (2001) The ATMChk2-Cdc25A Checkpoint Pathway Guards against Radioresistant DNA Synthesis. Nature, 410, 842-847. https://doi.org/10.1038/35071124

[14] Swift, M., Reitnauer, P.J., Morrell, D. and Chase, C.L. (1987) Breast and Other Cancers in Families with Ataxia-Telangiectasia. The New England Journal of Medicine, 316, 1289-1294. https://doi.org/10.1056/NEJM198705213162101

[15] Morrell, D., Cromartie, E. and Swift, M. (1986) Mortality and Cancer Incidence in 263 Patients with Ataxia-Telangiectasia. Journal of the National Cancer Institute, 77, 89-92.

[16] Gotoff, S.P., Amirmokri, E. and Liebner, E.J. (1967) Ataxia Telangiectasia. Neoplasia, untoward Response to X-Irradiation, and Tuberous Sclerosis. American Journal of Diseases of Children, 114, 617-625. https://doi.org/10.1001/archpedi.1967.02090270073006

[17] Kastan, M.B. and Bartek, J. (2004) Cell-Cycle Checkpoints and Cancer. Nature, 432, 
316-323. https://doi.org/10.1038/nature03097

[18] Bartek, J., Lukas, C. and Lukas, J. (2004) Checking on DNA Damage in S Phase. Nature Reviews Molecular Cell Biology, 5, 792-804. https://doi.org/10.1038/nrm1493

[19] Bell, D.W., et al. (1999) Heterozygous Germ Line hCHK2 Mutations in Li-Fraumeni Syndrome. Science, 286, 2528-2531. https://doi.org/10.1126/science.286.5449.2528

[20] Banin, S., et al. (1998) Enhanced Phosphorylation of p53 by ATM in Response to DNA Damage. Science, 281, 1674-1677. https://doi.org/10.1126/science.281.5383.1674

[21] Donehower, L.A., et al. (1992) Mice Deficient for p53 Are Developmentally Normal But Susceptible to Spontaneous Tumours. Nature, 356, 215-221. https://doi.org/10.1038/356215a0

[22] Baker, S.J., et al. (1989) Chromosome 17 Deletions and p53 Gene Mutations in Colorectal Carcinomas. Science, 244, 217-221. https://doi.org/10.1126/science.2649981

[23] Malkin, D., et al. (1990) Germ Line p53 Mutations in a Familial Syndrome of Breast Cancer, Sarcomas, and Other Neoplasms. Science, 250, 1233-1238. https://doi.org/10.1126/science.1978757

[24] Rothkamm, K., Balroop, S., Shekhdar, J., Fernie, P. and Goh, V. (2007) Leukocyte DNA Damage after Multi-Detector Row CT: A Quantitative Biomarker of LowLevel Radiation Exposure. Radiology, 242, 244-251.

https://doi.org/10.1148/radiol.2421060171

[25] Berrington de Gonzalez, A., et al. (2009) Projected Cancer Risks from Computed Tomographic Scans Performed in the United States in 2007. Archives of Internal Medicine, 169, 2071-2077. https://doi.org/10.1001/archinternmed.2009.440

[26] Smith-Bindman, R., et al. (2009) Radiation Dose Associated with Common Computed Tomography Examinations and the Associated Lifetime Attributable Risk of Cancer. Archives of Internal Medicine, 169, 2078-2086. https://doi.org/10.1001/archinternmed.2009.427

[27] Pierce, D.A. and Preston, D.L. (2000) Radiation-Related Cancer Risks at Low Doses among Atomic Bomb Survivors. Radiation Research, 154, 178-186. https://doi.org/10.1667/0033-7587(2000)154[0178:RRCRAL]2.0.CO;2

[28] Preston, D.L., Pierce, D.A., Shimizu, Y., Ron, E. and Mabuchi, K. (2003) Dose Response and Temporal Patterns of Radiation-Associated Solid Cancer Risks. Health Physics, 85, 43-46. https://doi.org/10.1097/00004032-200307000-00010

[29] Preston, D.L., et al. (2007) Solid Cancer Incidence in Atomic Bomb Survivors: 1958-1998. Radiation Research, 168, 1-64. https://doi.org/10.1667/RR0763.1

[30] Beels, L., et al. (2012) Dose-Length Product of Scanners Correlates with DNA Damage in Patients Undergoing Contrast CT. European Journal of Radiology, 81, 1495-1499.

[31] Jost, G., et al. (2009) The Influence of X-Ray Contrast Agents in Computed Tomography on the Induction of Dicentrics and Gamma-H2AX Foci in Lymphocytes of Human Blood Samples. Physics in Medicine \& Biology, 54, 6029-6039. https://doi.org/10.1088/0031-9155/54/20/001

[32] Kuefner, M.A., et al. (2010) Effect of CT Scan Protocols on X-Ray-Induced DNA Double-Strand Breaks in Blood Lymphocytes of Patients Undergoing Coronary CT Angiography. European Radiology, 20, 2917-2924. https://doi.org/10.1007/s00330-010-1873-9

[33] Moller, P. (2005) Genotoxicity of Environmental Agents Assessed by the Alkaline Comet Assay. Basic \& Clinical Pharmacology \& Toxicology, 96, 1-42. 
[34] Ostling, O. and Johanson, K.J. (1984) Microelectrophoretic Study of RadiationInduced DNA Damages in Individual Mammalian Cells. Biochemical and Biophysical Research Communications, 123, 291-298.

[35] Ostling, O. and Johanson, K.J. (1987) Bleomycin, in Contrast to Gamma Irradiation, Induces Extreme Variation of DNA Strand Breakage from Cell to Cell. International Journal of Radiation Biology and Related Studies in Physics, Chemistry, and Medicine, 52, 683-691. https://doi.org/10.1080/09553008714552201

[36] Baskerville-Abraham, I.M., et al. (2009) Development of an Ultraperformance Liquid Chromatography/Mass Spectrometry Method to Quantify Cisplatin 1,2 Intrastrand Guanine-Guanine Adducts. Chemical Research in Toxicology, 22, 905-912.

[37] Svilar, D., Vens, C. and Sobol, R.W. (2012) Quantitative, Real-Time Analysis of Base Excision Repair Activity in Cell Lysates Utilizing Lesion-Specific Molecular Beacons. Journal of Visualized Experiments, No. 66, 4168. https://doi.org/10.3791/4168

[38] Fry, R.C., et al. (2008) Genomic Predictors of Inter-Individual Differences in Response to DNA Damaging Agents. Genes \& Development, 22, 2621-2626. https://doi.org/10.1101/gad.1688508

[39] Nagel, Z.D., Chaim, I.A. and Samson, L.D. (2014) Inter-Individual Variation in DNA Repair Capacity: A Need for Multi-Pathway Functional Assays to Promote Translational DNA Repair Research. DNA Repair, 19, 199-213.

[40] Nagel, Z.D., et al. (2017) DNA Repair Capacity in Multiple Pathways Predicts Chemoresistance in Glioblastoma Multiforme. Cancer Research, 77, 198-206. https://doi.org/10.1158/0008-5472.CAN-16-1151

[41] Nagel, Z.D., et al. (2014) Multiplexed DNA Repair Assays for Multiple Lesions and Multiple Doses via Transcription Inhibition and Transcriptional Mutagenesis. Proceedings of the National Academy of Sciences of the United States, 111, 1823-1832. https://doi.org/10.1073/pnas.1401182111

[42] Weingeist, D.M., et al. (2013) Single-Cell Microarray Enables High-Throughput Evaluation of DNA Double-Strand Breaks and DNA Repair Inhibitors. Cell Cycle, 12, 907-915. https://doi.org/10.4161/cc.23880

[43] Garty, G., et al. (2010) The RABIT: A Rapid Automated Biodosimetry Tool for Radiological Triage. Health Physics, 98, 209-217. https://doi.org/10.1097/HP.0b013e3181ab3cb6

[44] Turner, H.C., et al. (2014) The RABiT: High-Throughput Technology for Assessing Global DSB Repair. Radiation and Environmental Biophysics, 53, 265-272. https://doi.org/10.1007/s00411-014-0514-0

[45] Gowan, S.M., et al. (2007) Application of Meso Scale Technology for the Measurement of Phosphoproteins in Human Tumor Xenografts. Assay and Drug Development Technologies, 5, 391-401. https://doi.org/10.1089/adt.2006.044

[46] Liang, M., et al. (2007) Detection of High- and Low-Affinity Antibodies against a Human Monoclonal Antibody Using Various Technology Platforms. Assay and Drug Development Technologies, 5, 655-662. https://doi.org/10.1089/adt.2007.089

[47] Cao, L., et al. (2008) Addiction to Elevated Insulin-Like Growth Factor I Receptor and Initial Modulation of the AKT Pathway Define the Responsiveness of Rhabdomyosarcoma to the Targeting Antibody. Cancer Research, 68, 8039-8048. https://doi.org/10.1158/0008-5472.CAN-08-1712

[48] Fichorova, R.N., et al. (2008) Biological and Technical Variables Affecting Immunoassay Recovery of Cytokines from Human Serum and Simulated Vaginal Fluid: A Multicenter Study. Analytical Chemistry, 80, 4741-4751. https://doi.org/10.1021/ac702628q 
[49] Begley, U., et al. (2013) A Human tRNA Methyltransferase 9-Like Protein Prevents Tumour Growth by Regulating LIN9 and HIF1-Alpha. EMBO Molecular Medicine, 5, 366-383. https://doi.org/10.1002/emmm.201201161

[50] Lobrich, M., et al. (2005) In Vivo Formation and Repair of DNA Double-Strand Breaks after Computed Tomography Examinations. Proceedings of the National Academy of Sciences of the United States, 102, 8984-8989.

https://doi.org/10.1073/pnas.0501895102

[51] Steinberg, K.K. (2001) Ethical Challenges at the Beginning of the Millennium. Statistics in Medicine, 20, 1415-1419. https://doi.org/10.1002/sim.678

\section{Supplementary Materials}

Supplementary Tables, Figure Legend and Figures.

Submit or recommend next manuscript to SCIRP and we will provide best service for you:

Accepting pre-submission inquiries through Email, Facebook, LinkedIn, Twitter, etc. A wide selection of journals (inclusive of 9 subjects, more than 200 journals)

Providing 24-hour high-quality service

User-friendly online submission system

Fair and swift peer-review system

Efficient typesetting and proofreading procedure

Display of the result of downloads and visits, as well as the number of cited articles Maximum dissemination of your research work

Submit your manuscript at: http://papersubmission.scirp.org/

Or contact abb@scirp.org 